\title{
Comparación entre el cuestionario "ICIQ-UI Short Form" y el "King's Health Questionnaire" como instrumentos de evaluación de la incontinencia urinaria en mujeres
}

\author{
Espuña Pons $\mathrm{M}^{1}$, Castro Díaz $\mathrm{D}^{2}$, Carbonell $\mathrm{C}^{3}$, Dilla $\mathrm{T}^{4}$. \\ ${ }^{1}$ Institut Clínic de Ginecología, Obstetricia iNeonatología. Hospital Clínic. Barcelona University. ${ }^{2}$ Hospital \\ Universitario de Canarias. ${ }^{3}$ ABS Via Roma. ${ }^{4}$ Department of Clinical Research, Lilly S.A., Madrid.
}

Actas Urol Esp. 2007;31(5):502-510

\section{RESUMEN}

COMPARACIÓN ENTRE EL CUESTIONARIO “ICIQ-UI SHORT FORM” Y EL “KING'S HEALTH QUESTIONNAIRE” COMO INSTRUMENTOS DE EVALUACIÓN DE LA INCONTINENCIA URINARIA EN MUJERES

Introducción: En nuestro país existen diferentes cuestionarios para la evaluación de la Incontinencia Urinaria (IU) en la práctica clínica, desde el punto de vista de la paciente. El "King's Health Questionnaire" (KHQ) y el "International Consultation on Incontinence Questionnaire-Urinary Incontinence Short Form” (ICIQ-UI SF) son los más utilizados. El presente estudio tuvo por objetivo comparar la utilidad clínica de KHQ y el ICIQ-UI SF respecto a la prueba urodinámica.

Material y Métodos: Estudio transversal de 116 mujeres que completaron el ICIQ-UI SF, el KHQ y la prueba urodinámica, recibiendo un diagnóstico de tipo de IU según cada una de estas 3 pruebas. Se estudiaron la sensibilidad y especificidad de la dimensión de síntomas para el diagnóstico del tipo de IU del ICIQ-UI SF y del KHQ respecto a la prueba urodinámica. Además, se analizó la correlación existente entre ambas medidas, ICIQ-UI SF y KHQ.

Resultados: La edad media fue de 54 años (DE= 13,99). La puntuación media del KHQ fue 39,93 $(22,11)$ y la del ICIQ-UI SF $13,76(4,11)$, presentando ambos cuestionarios una correlación moderada $(r=0,6 ; p<0,001)$. Los porcentajes de pacientes con síntomas sugestivos de IU de Esfuerzo (IUE), IU de Urgencia (IUU) e IU Mixta (IUM) fueron respectivamente 33,7, 17,3 y 49 para el KHQ; 40,4, 15,4 y 44,2 para el ICIQ-UI SF. La distribución de las pacientes según las observaciones en el estudio urodinámico fue: IUE $41,3 \%$, IUU 20,2\%, IUM $26,9 \%$ y un $11,5 \%$ otros diagnósticos. La sensibilidad y especificidad de ambos cuestionarios fueron muy similares, pero la factibilidad fue peor para el KHQ (7,76\% no lo completaron) que para el ICIQ-UI SF (2,59\% no lo completaron).

Conclusiones: Por su mejor factibilidad parece aconsejable el uso preferencial del ICIQ-UI SF frente al KHQ a la hora de evaluar la IU en la práctica clínica.

Palabras clave: Incontinencia de orina. Diagnóstico. Cuestionarios.

\begin{abstract}
COMPARISON BETWEEN THE “ICIQ-UI SHORT FORM" QUESTIONNAIRE AND THE “KING'S HEALTH QUESTIONNAIRE” AS ASSESSMENT TOOLS OF URINARY INCONTINENCE AMONG WOMEN.

Introduction: In our country there are a few available instruments to diagnose urinary incontinence (UI) from the patient's perspective. The King's Health Questionnaire (KHQ) and the "International Consultation on Incontinence Questionnaire-Urinary Incontinence Short Form" (ICIQ-UI SF) are the most widespread among that. The present study aimed to compare the clinical utility between $\mathrm{KHQ}$ and ICIQ-UI SF with regard to the urodynamic test.

Material and Methods: Cross-sectional study performed in 116 women who completed the ICIQ-UI SF, the KHQ and the urodynamic test and were diagnosed according to each test. Sensibility and specificity values of symptom dimension of the ICIQ$\mathrm{UI} \mathrm{SF}$ and the KHQ were analyzed with regard to the urodynamic test. In addition, correlation scores between the both compared measures were calculated.

Results: Mean age (SD) of women was 54 years (SD=13.99). KHQ and ICIQ-UI SF mean scores were 39.93 (22.11) and 13.76 (4.11), respectively. Correlation between both measures was moderated ( $r=0.6 ; p<0.001)$. Percentages of pts with symptoms suggesting Stress UI (SUI), Urge UI (UUI) and Mixed UI (MUI) according to each instrument were: 33.7, 17.3 \& 49 (KHQ); $40.4,15.4$ \& 44.2 (ICIQ-UI SF). Patients' distribution according to urodynamic test was: SUI $41.3 \%$, UUI $20.2 \%$, MUI $26.9 \%$ and $11.5 \%$ with other diagnosis. Sensibility and specificity values of both questionnaires were very similar, but feasibility was worse for the KHQ (7.76\% of pts did not complete the questionnaire) than for the ICIQ-UI SF ( $2.59 \%$ did not complete the questionnaire).

Conclusions: Because of its better feasibility, clinical use of ICIQ-UI SF is recommended against KHQ for UI evaluation.
\end{abstract}

Keywords: Urinary incontinence. Diagnostic. Questionnaires. 
$\mathrm{L}$ a International Continence Society (ICS), define la incontinencia urinaria (IU) como cualquier pérdida involuntaria de orina ${ }^{1}$. La sintomatología asociada a estas pérdidas es muy variada y de alta prevalencia en la población general de mujeres. Así, se estima que entre el 10\% y el 50\% de las mujeres padecen este problema a lo largo de su vida ${ }^{2,3}$. Esta importancia epidemiológica parece confirmarse en estudios más recientes como el llevado a cabo en varios países europeos (Alemania, España, Francia y Reino Unido) por Hunskaar et $\mathrm{al}^{4}$. En esta investigación, después de evaluar a un total de 17.080 mujeres, se encontró que el 35\% habían experimentado algún tipo de pérdida de orina en los últimos 30 días y tan sólo una cuarta parte de las mismas habían consultado a su médico por este motivo. De forma específica, en nuestro país las cifras se sitúan en torno al 20\% y el 50\%.

A partir de su manifestación sintomática, la IU se puede clasificar en tres tipos fundamentales: Incontinencia Urinaria de Esfuerzo (IUE), que refiere la pérdida involuntaria de orina producida en la realización de una actividad física o en actos simples tales como el toser o estornudar; la Incontinencia Urinaria de Urgencia (IUU) definida como la pérdida involuntaria de orina que se acompaña o que es precedida de forma inmediata por una intensa sensación de urgencia y finalmente, Incontinencia Urinaria Mixta (IUM) que incluye sintomatología de las anteriores tipologías $^{1}$. Estas clasificaciones nosológicas son de interés debido a que permiten decidir qué terapia es más adecuada para cada caso particular.

Por otro lado, a pesar de que la IU no constituye un riesgo vital para la persona, sí conlleva un evidente impacto en su calidad de vida. Esto es así, debido a las implicaciones no sólo físicas, sino también psicológicas y sociales de esta patología. Como consecuencia, la Internacional Consultation on Incontinente (ICI) defiende que el impacto en las pacientes no sólo debe valorarse a partir de la presencia o no de determinados síntomas sino también a través de la evaluación del grado de afectación de la calidad de vida de las pacientes $^{5}$. Así pues, debería ser evaluado en cada caso el tipo de síntomas asociados a la IU, la gravedad de los mismos y el grado de afectación que producen dependiendo de las caracte- rísticas individuales de cada paciente. Debido a esto, se considera de especial interés el desarrollo de instrumentos simples que permitan discernir desde el primer momento el tipo de IU así como el grado de afectación que sufre la paciente con el objeto de ajustar en la medida de lo posible el tratamiento ${ }^{6}$. Para la consecución de este objetivo y siguiendo las recomendaciones de la tercera edición de la ICI, es necesario conocer la opinión del paciente a través de la autocumplimentación de cuestionarios de evaluación fiables y válidos ${ }^{7}$.

Actualmente, en la práctica clínica varios son los instrumentos disponibles con este doble propósito (descriptivo y diagnóstico). Entre ellos, el King's Health Questionnaire (KHQ) y el ICIQ-UI SF son probablemente los más utilizados disponiendo de versiones adaptadas a nuestro país ${ }^{8,9}$.

La importancia de estos instrumentos clínicos reside, fundamentalmente, en la capacidad de los mismos para explicar y describir el perjuicio que la IU provoca en la calidad de vida (CV) de la mujer y también en su capacidad para discernir entre los distintos tipos de IU. Esto último puede conocerse a través del ajuste diagnóstico de estas pruebas en relación a otra considerada como de referencia en el ámbito de la IU como es la prueba Urodinámica.

En nuestro estudio se pretende conocer en primer lugar, la relación existente entre el KHQ y el ICIQ-UI SF en cuanto su valor descriptivo del impacto de la IU en la CV de la paciente y en segundo lugar, comparar la utilidad clínica de KHQ y el ICIQ-UI SF respecto a la prueba urodinámica, estudiando la sensibilidad y especificidad de los dos instrumentos para el diagnóstico de cada tipo de IU, de Esfuerzo, de Urgencia y Mixta.

\section{PACIENTES Y MÉTODOS}

Estudio observacional, transversal en el que se incluyeron un total de 116 pacientes consecutivas con incontinencia urinaria usuarias de dos centros sanitarios especializados: la Unidad de Suelo Pélvico del Hospital Clinic de Barcelona y el Departamento de Urología del Hospital Universitario de Canarias en Tenerife. Sobre esta muestra se presentaron previamente otros resultados del estudio llevado a cabo por Espuña et $\mathrm{al}^{10}$. 
Los criterios para la selección de los participantes han sido los siguientes:

1. Mujer mayor de 18 años

2. Ausencia de infección urinaria determinada mediante análisis (dipstick)

3. Puntuación en el ICIQ-UI SF para la suma de los apartados 3 y 4 mayor que 0 e igualmente, puntuación mayor que 0 en el apartado 5. Es decir que mediante este cuestionario autoadministrado, se objetive que la mujer con sintomas de IU, percibe que le molestan.

Ademas de los criterios referidos, no se incluyen en el estudio pacientes con enfermedades neurológicas degenerativas, psicosis, antecedentes traumáticos severos y oncológicos. De igual forma, no forman parte del estudio aquellas pacientes con prolapso genital severo, mujeres embarazadas, aquellas que se encuentren en periodo de postparto ( $<6$ meses), y por último, pacientes que se encuentren bajo tratamiento en el momento del estudio.

Todas las pacientes completaron la versión española del King`s Health Questionnaire (KHQ), el ICIQ-UI SF y de igual forma, realizaron la prueba urodinámica.

$\mathrm{El} \mathrm{KHQ}{ }^{11}$ es un instrumento autoadministrado de evaluación específico de la calidad de vida en mujeres con IU. Consta de 21 items distribuidos en 9 dimensiones: Percepción del Estado de Salud General-ESG (1 item), Afectación por Problemas Urinarios-APU (1 ítem), Limitaciones en las Actividades Cotidianas-LAC (2 ítems), Limitaciones Sociales-LS (2 items), Limitaciones Físicas-LF (2 items), Relaciones Personales-RP (3 items), Emociones-E (3 items), Sueño/EnergíaSE (2 items) e Impacto de la IU-ImIU (5 items). Cada ítem tiene una escala de respuesta tipo Likert con 4 posibles respuestas. El rango de puntuaciones de cada dimensión va de 0 (menor impacto de la IU y por tanto mejor calidad de vida) a 100 (mayor impacto, peor calidad de vida). Este cuestionario permite obtener un valor global de la calidad de vida de la paciente con IU (Puntuación Global-PG) y otro específico para cada dimensión dentro de una escala con la siguiente amplitud:

0: Mejor calidad de vida posible - 100: Peor calidad de vida posible.
La versión española de este cuestionario ha demostrado tener adecuados índices de validez y fiabilidad para evaluar la calidad de vida de las mujeres con diferentes tipos de $\mathrm{IU}^{8}$.

Para poder orientar el tipo de incontinencia que padece cada participante, este cuestionario cuenta con un apartado específico de síntomas urinarios (apartado $\mathrm{n}^{\circ}$ 3, Tabla 1) que no es tenido en cuenta para el cálculo de la puntuación global y la de las dimensiones pero que sí es de interés para la clasificación de la IU. En este apartado, la paciente debe señalar sólo aquellos sintomas asociados a la IU que padece y también, valorar la intensidad de esta afectación como poca, moderada o mucha. De esta forma, a partir de las respuestas de los sujetos a este apartado, se procedió a la clasificación de las pacientes según los síntomas, teniendo en cuenta los siguientes criterios:

1. Se considera que una paciente tiene síntomas sugestivos de IUE si señala alguna de las respuestas (cualquiera de los grados de afectación) de la pregunta 4 de este apartado ("escape de orina por actividad física") y no indica alguna de las respuestas de la pregunta 3 ("escape de orina asociado a un fuerte deseo de orinar").

2 . Se considera que una paciente tiene sintomas de IUU si señala alguna de las respuestas (cualquiera de los grados de afectación) de la pregunta 3 de este apartado ("escape de orina asociado a un fuerte deseo de orinar") y no indica alguna de las respuestas de la pregunta 4 ("escape de orina por actividad física”).

3. IUM: se considera que una paciente tiene sintomas de IUM si señala alguna de las respuestas (en cualquiera de los grados de afectación) de las preguntas 3 y 4 de este apartado (escape de orina asociado a un fuerte deseo de orinar y escape de orina por actividad física).

El cuestionario ICIQ-UI SF consiste en 3 items (Frecuencia, Cantidad e Impacto) y 8 preguntas más relacionadas con los síntomas orientativos del tipo de IU. Estas últimas preguntas no se contemplan a la hora de obtener un índice global del ICIQ-UI SF sino que su valor radica en la descripción de las circunstancias que pueden provocar la UI a la paciente ${ }^{12}$. De esta forma, la puntuación total de cada paciente se calcula con la 
Tabla 1

Preguntas relacionadas con los sintomas urinarios en el cuestionario King's Health Questionnaire (KHQ). Apartado 3 del cuestionario

¿Hasta qué punto le afectan? Nos gustaría saber cuáles son sus problemas urinarios y hasta qué punto le afectan. De la lista siguiente elija SÓLO AQUELLOS PROBLEMAS que usted tenga en la actualidad. DEJE SIN CONTESTAR los que no correspondan a su caso. (Para contestar marque con una cruz).

\begin{tabular}{llll}
\hline Un poco & Moderadamente & Mucho \\
\hline
\end{tabular}

FRECUENCIA: ir al baño muy a menudo

URGENCIA: un fuerte deseo de orinar dificil de controlar

INCONTINENCIA POR URGENCIA: escape de orina asociado

a un fuerte deseo de orinar

INCONTINENCIA POR ESFUERZO: escape de orina por actividad física

(ej., toser, estornudar, correr)

ENURESIS NOCTURNA: mojar la cama durante la noche

INCONTINENCIA EN EL ACTO SEXUAL: escape de orina durante

el acto sexual (coito)

INFECCIONES FRECUENTES EN LAS VÍAS URINARIAS

DOLOR EN LA VEJIGA

Dificultad AL ORINAR

OTRO PROBLEMA URINARIO (Especificar):

suma de los 3 primeros ítems con un rango que oscila entre 0 y 21 puntos. La versión española de este cuestionario ${ }^{9}$ ha demostrado tener unos indices de fiabilidad, consistencia interna y validez no sólo en la descripción del impacto de la IU en la vida de los pacientes sino también para poder hacer una orientación del tipo de IU, en la práctica clínica. Para este último propósito, el cuestionario incluye, como en el caso de KHQ, un apartado específico (Tabla 2). Las pacientes en este caso deben señalar todos aquellos sintomas relacionados con la IU que sean aplicables a su caso en particular. A partir de las respuestas obtenidas, se procedió a su clasificación diagnóstica siguiendo los siguientes criterios:

- Se considera que un paciente tiene sintomas sugestivos de IUE si se contemplan como afirmativas al menos una de las siguientes preguntas: pérdida de orina mientras tose o estornuda, pérdida de orina cuando se realiza un esfuerzo físico o ejercicio. Además de señalar al menos una de las anteriores, la pregunta referida a las pérdidas de orina antes de llegar al baño no debe ser señalada.

\section{Tabla 2}

Preguntas relacionadas con los sintomas urinarios en el cuestionario de incontinencia urinaria "International Continence Questionnaire- short form (ICIQ-UI SF)

¿Cuándo pierde orina? (Señale todo lo que le pasa a usted) Nunca pierde orina

Pierde orina antes de llegar al WC

Pierde orina cuando tose o estornuda

Pierde cuando duerme

Pierde orina cuando hace esfuerzos físicos/ejercicio

Pierde orina al acabar de orinar y ya se ha vestido

Pierde orina sin un motivo evidente

Pierde orina de forma continua

- Se considera que una paciente tiene sintomas sugestivos de IUU cuando refiere problemas de pérdida de orina antes de llegar al baño y no señala ningún ítem específico de IUE

- Se considera que un paciente sufre IUM cuando indica problemas de pérdida de orina antes de llegar al baño y alguna de los síntomas asociados a IUE. 
Además, al completar los anteriores cuestionarios, todas las pacientes fueron sometidas a un estudio urodinámico que comprendía las siguientes pruebas: flujometría con medición del residuo postmiccional, cistometría de llenado y la medición de la presión-flujo durante la micción (cistometría de vaciado vesical). Dentro del proceso diagnóstico del tipo de IU, este conjunto de pruebas forman parte de la práctica clínica habitual de los centros especializados donde se llevó a cabo este estudio. El estudio urodinámico se realizó de acuerdo a los criterios definidos en el protocolo de actuación de la Internacional Continente Society $(\mathrm{ICS})^{13}$. De acuerdo al estudio urodinámico llevado a cabo, los pacientes son clasificados en 5 grupos:

1. Pacientes con incontinencia urodinámica de esfuerzo (IUEU): si en la cistomanometría de llenado se produce pérdida de orina durante el aumento de la presión abdominal (toser con vejiga llena, en posición ortostática) y no se objetiva ninguna contracción involuntaria del detrusor.

2. Pacientes con hiperactividad del detrusor (HD): si se observan contracciones involuntarias del detrusor asociados con una sensación de urgencia durante la cistomanometría de llenado o durante la ejecución de las maniobras de provocación.

3. Pacientes con HD e IUEU.

4. Pacientes con estudio urodinámico normal: si no se observa ninguna anormalidad sintomática como las referidas anteriormente durante el estudio urodinámico.

5. Pacientes con otras observaciones en el estudio urodinámico (como disfunción del vaciado vesical).

Por último, los especialistas que realizaban esta clasificación diagnóstica basada en la prueba urodinámica desconocían los resultados de la clasificación de las pacientes basada en los dos cuestionarios (KHQ e ICIQ-UI SF).

\section{ANÁLISIS ESTADÍSTICO}

En primer lugar, se describe la muestra participante en este estudio a través del cálculo de los porcentajes, media y desviación estándar de las variables sociodemográficas y clinicas de mayor interés.

A continuación, se describen las pruebas King's Health Questionnaire e ICIQ-UI SF a través de las puntuaciones de los pacientes en cada dimensión y en el conjunto de las mismas.
Tras el análisis descriptivo, se procede a analizar de forma comparativa la sensibilidad y especificidad del KHQ y el ICIQ-UI SF respecto a la prueba urodinámica. Junto a estos indicadores, también se calcula el coeficiente "Likelihood Ratio" (LR) ya que ayuda a definir el grado de utilidad de una prueba diagnóstica.

\section{$\mathrm{LR}=$ sensibilidad / 1 - especificidad}

De esta forma, consideramos que una buena prueba diagnóstica debe tener una alta sensibilidad y un alto valor positivo en el cociente de probabilidad positivo ${ }^{14}$.

En este análisis comparativo entre los cuestionarios y la prueba urodinámica sólo se han tenido en cuenta los datos procedentes de los sujetos que podían ser diagnosticados de forma diferencial (IUE; IUU; IUM) en cada una de las tres pruebas citadas. De esta forma, de los 116 pacientes iniciales, serán 104 los que configurarán el tamaño muestral en estos apartados.

Para conocer la relación existente entre las puntuaciones en el King's Health Questionnaire y en el ICIQ-UI SF se estudiará la correlación de las puntuaciones globales de ambas pruebas y también de forma específica la del ítem 3 del ICIQ-UI SF respecto a la puntuación global del KHQ.

El análisis estadístico se realizó utilizando el paquete de software estadístico SPSS 14.0 para Windows. En todas las pruebas se consideró un nivel de significación alfa $\alpha$ del 0,05.

\section{RESULTADOS}

La edad media de las 116 pacientes participantes en este estudio es aproximadamente de 54 años (DS= 13,99) con unos valores medios de Índice de Masa Corporal de 28,33 (DS= 7,55). Un porcentaje muy alto de estas mujeres se encuentra trabajando $(80,4 \%)$ bien dentro de sus hogares $(40,2 \%)$ o bien como empleadas en un trabajo remunerado $(40,2 \%)$. En cuanto al historial clínico de las pacientes, el 40,7\% habian sido diagnosticadas con anterioridad de problemas de IU y el 8,6\% habían sido intervenidas con una técnica antiincontinencia. Por último, el 64,7\% de estas pacientes estaban tomando algún tipo de fármaco para un problema médico; en un 4,3\% se trataba de un fármaco antimuscarínico (Tabla 3). 
Tabla 3

Características demográficas y antecedentes generales de la muestra estudiada

\begin{tabular}{|c|c|c|c|c|}
\hline \multicolumn{5}{|c|}{ Descripción de la muestra del estudio $(\mathrm{N}=116)$} \\
\hline Edad media (DE) & & 54,32 & & \\
\hline Situación laboral, n (\%) & $\begin{array}{l}\text { Ama de casa } \\
45(40,2 \%)\end{array}$ & $\begin{array}{l}\text { En activo } \\
45(40,2 \%)\end{array}$ & $\begin{array}{l}\text { Jubilada } \\
18(16,1 \%)\end{array}$ & $\begin{array}{l}\text { Otra } \\
8(3,5 \%)\end{array}$ \\
\hline Media IMC (DS) & \multicolumn{4}{|c|}{$28,33(7,55)$} \\
\hline Diagnóstico previo de IU, n (\%) & \multicolumn{4}{|c|}{$46(40,7 \%)$} \\
\hline $\begin{array}{l}\text { Antecedentes de cirugía abdominal, n (\%) } \\
\text { Cirugia uroginecológica. }\end{array}$ & \multicolumn{4}{|c|}{$59(50,9 \%)$} \\
\hline Histerectomía (abdominal o vaginal), n (\%) & \multicolumn{4}{|c|}{$17(14,7 \%)$} \\
\hline Intervención vaginal por prolapso genital, $\mathrm{n}(\%)$ & \multicolumn{4}{|c|}{$5(4,3 \%)$} \\
\hline Intervención correctiva de la IU, n (\%) & \multicolumn{4}{|c|}{$10(8,6 \%)$} \\
\hline Intervención ano-rectal, n (\%) & \multicolumn{4}{|c|}{$5(4,3 \%)$} \\
\hline \multicolumn{5}{|l|}{ Actualmente, tomando algún tipo de medicación } \\
\hline (para cualquier problema de salud) & \multicolumn{4}{|c|}{$75(64,7 \%)$} \\
\hline \multicolumn{5}{|c|}{ Fármacos relacionados con los sintomas urinarios } \\
\hline Antimuscarínícos & \multicolumn{4}{|c|}{$5(4,3 \%)$} \\
\hline Antidepresivos & \multicolumn{4}{|c|}{$16(13,8 \%)$} \\
\hline Diuréticos & \multicolumn{4}{|c|}{$7(6 \%)$} \\
\hline
\end{tabular}

En el análisis comparativo entre los cuestionarios KHQ, ICIQ-UI SF y la prueba Urodinámica y siguiendo el criterio de selección de los casos definido en el apartado anterior cuyo objetivo prioritario es asegurar la rigurosidad de los resultados, 13 pacientes han sido excluidas: una paciente que no puede ser incluida en ninguno de los grupos según el estudio urodinámico $(0,86 \%)$, tres según el ICIQ-UI SF $(2,59 \%)$ y por último, nueve pacientes según el cuestionario KHQ $(7,76 \%)$.

En cuanto a los resultados del cuestionario $\mathrm{KHQ}$, la puntuación media de la escala global $(\mathrm{n}=98)$ es de 39,93 $(22,11)$. La media (DS) de los valores obtenidos en cada una de las nueve dimensiones que lo configuran se presentan de forma gráfica en la Figura 1.

La puntuación media del cuestionario ICQ-UI $\mathrm{SF}(\mathrm{n}=101)$ es de $13,76(4,11)$ siendo 4 el valor mínimo y 21 el máximo.

La correlación entre las puntuaciones globales de estos dos cuestionarios (correlación de Pearson) es de 0,6 ( $\mathrm{p}=0,001)$. Por su parte, la relación entre el ítem 3 del ICIQ-UI SF que evalúa el impacto de la IU en la vida de la persona con una escala de intervalo desde el 0 (no afecta nada) a 10 (afecta mucho) y el valor global del KHQ también se evaluó con el mismo estadístico obteniendo un valor de $0,55(\mathrm{p}<0,001)$. Por

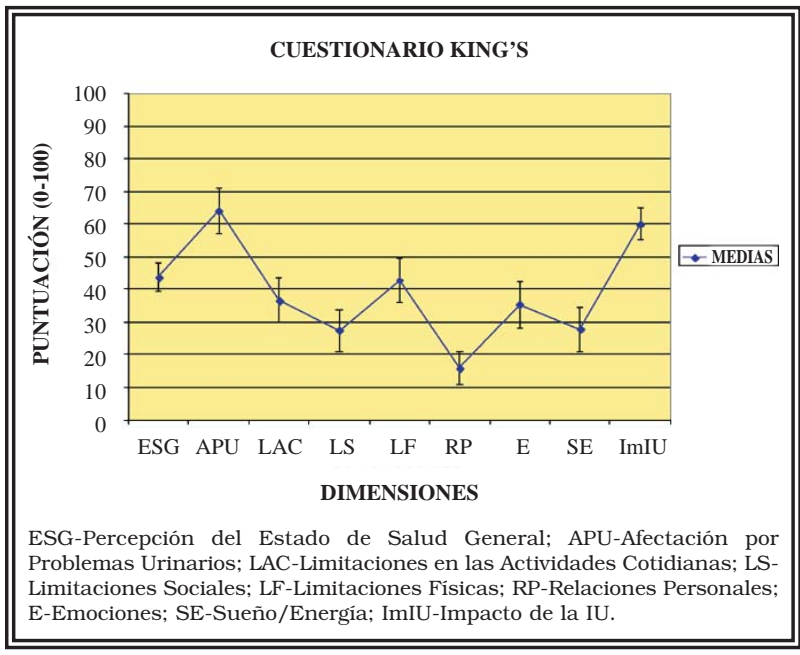

FIGURA 1. Valores obtenidos en cada una de las nueve dimensiones que configuran el King's Health Questionnaire.

último, este mismo ítem 3 del ICIQ-UI SF puesto en relación con la dimensión 2 del KHQ relativa al impacto de la IU en la calidad de vida del paciente tiene como resultado un valor de 0,57 $(\mathrm{p}<0,001)$.

La clasificación de los casos de IU realizada a partir de los criterios de las 3 pruebas diagnósticas (Tabla 4) ha sido la siguiente:

Siguiendo los síntomas referidos por las pacientes en el cuestionario $\mathrm{KHQ}, 35$ mujeres presentaban síntomas sugestivos de IUE, 18 de IUU, y finalmente, 51 casos tienen IUM. 
Tabla 4

Relación entre la clasificación en grupos sintomáticos según el King’s Health Questionnaire (KHQ) y el cuestionario de incontinencia urinaria "International Continence Questionnaire- short form (ICIQ-UI SF)

\begin{tabular}{|c|c|c|c|c|c|c|c|}
\hline \multicolumn{8}{|c|}{ CLASIFICACIONES SINTOMÁTICAS vS ESTUDIO URODINÁMICO } \\
\hline & & \multicolumn{6}{|c|}{ Diagnóstico Urodinámico } \\
\hline & & \multicolumn{2}{|c|}{ IUE } & \multicolumn{2}{|c|}{ IUU } & \multicolumn{2}{|c|}{ IUM } \\
\hline & & - & + & - & + & - & + \\
\hline \multirow[t]{2}{*}{ King's } & - & 51 & 18 & 75 & 11 & 42 & 11 \\
\hline & + & 10 & 25 & 8 & 10 & 34 & 17 \\
\hline \multirow[t]{3}{*}{ ICIQ-UI SF } & - & 43 & 19 & 76 & 12 & 48 & 10 \\
\hline & + & 18 & 24 & 7 & 9 & 28 & 18 \\
\hline & & 61 & 43 & 83 & 21 & 76 & 28 \\
\hline
\end{tabular}

Según los síntomas señalados por las mujeres en el ICIQ-UI SF, 42 pacientes podrían ser clasificadas como de padecer IUE, 16 referian síntomas sugestivos de IUU y 46 presentaban los dos tipos de sintomatología (IUM).

Por último, de acuerdo a los hallazgos del estudio urodinámico, se consideró que 43 pacientes tenían una IUE, 21 una IUU y 28 una IUM. En el resto de las mujeres, 12, el estudio urodinámico demostró una disfunción distinta a la IU, que no es tenido en cuenta en los análisis comparativos de los valores predictivos.

$\mathrm{El}$ estudio de las frecuencias de las coincidencias y disparidades diagnósticas entre los distintos instrumentos ha permitido conocer las puntuaciones relativas a la sensibilidad, especificidad y LR de los cuestionarios analizados para cada una de las tipologías comentadas de la IU respecto a la prueba urodinámica (Tabla 5 y Fig. 2). Como se puede observar, estos valores son similares en ambos instrumentos para cada una de las tipologías estudiadas de la IU.

\section{DISCUSIÓN}

La utilidad en la identificación y cuantificación del impacto de la IU en la calidad de vida de los pacientes así como el valor para el diagnóstico del tipo de IU de los cuestionarios KHQ y el ICIQ-UI SF ha sido resaltada en múltiples estu$\operatorname{dios}^{8,9,11}$. En esta misma línea, con los resultados obtenidos en nuestra investigación no parecen existir grandes diferencias entre los cuestionarios KHQ e ICIQ-UI SF en cuanto a los índices de sensibilidad, especificidad y valores predictivos si tomamos como referencia la evaluación urodinámica en el diagnóstico de las distintas tipologías de la IU que han sido analizadas (IUE, IUU, IUM). Esto es, tal y como se refleja en la Tabla 5 los dos cuestionarios tienen un valor diagnóstico similar. Si se representan de forma gráfica (Fig. 2), vemos que para las distintas tipologías de IU analizadas en ambos cuestionarios estos valores predictivos están situados muy próximos entre sí.

A pesar de esto, sí se producen diferencias importantes en la factibilidad de uso en la práctica clínica debidas no sólo al tamaño de cada

\section{Tabla 5}

Valor del King's Health Questionnaire (KHQ) y el cuestionario de incontinencia urinaria "International Continence Questionnaire-short form (ICIQ-UI SF) para la orientación diagnóstica del tipo de incontinencia urinaria en relación al diagnóstico urodinámico.

\begin{tabular}{llccc}
\hline \multicolumn{3}{c}{ VALORES DE LAS PRUEBAS DIAGNÓSTICAS } \\
\hline & & \multicolumn{3}{c}{ DIAGNÓSTICO } \\
& & URODINÁMICO \\
& & IUE & IUU & IUM \\
\hline KHQ & sensibilidad & 0,58 & 0,48 & 0,61 \\
& especificidad & 0,84 & 0,90 & 0,55 \\
& VPP & 0,71 & 0,56 & 0,33 \\
& VPN & 0,74 & 0,87 & 0,79 \\
ICIQ-IU SF & LR & 3,55 & 4,94 & 1,36 \\
& sensibilidad & 0,56 & 0,43 & 0,64 \\
& especificidad & 0,70 & 0,92 & 0,63 \\
& VPP & 0,57 & 0,56 & 0,39 \\
& VPN & 0,69 & 0,86 & 0,83 \\
& LR & 1,89 & 5,08 & 1,75 \\
\hline
\end{tabular}

KHQ-King's Health Questionnaire

ICIQ-UI SF-International Continence Questionnaire-short form. 


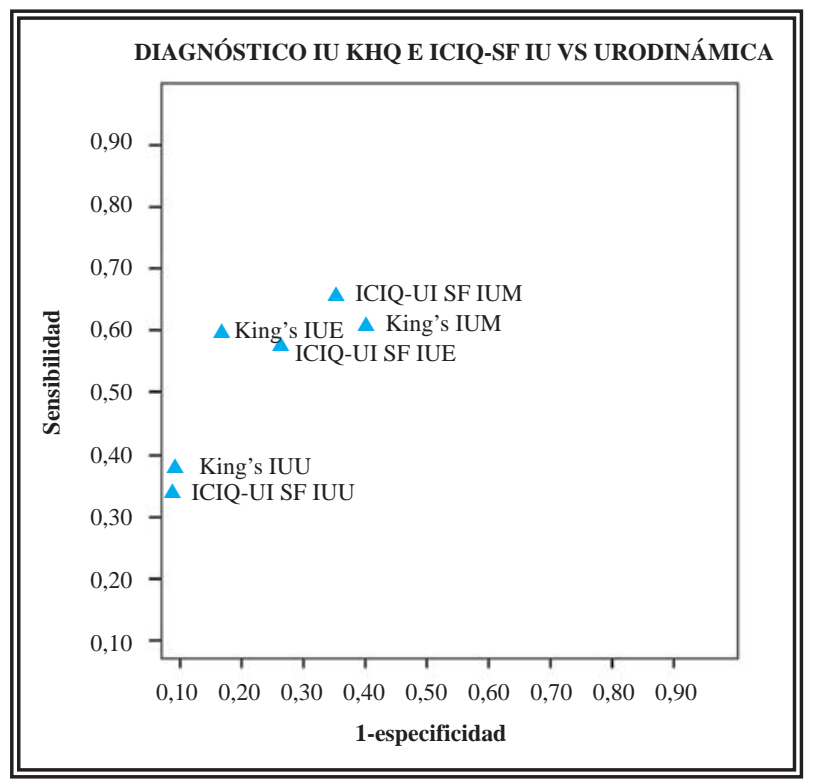

FIGURA 2. Relación entre los valores predictivos del King's Health Questionnaire (KHQ) y del cuestionario de incontinencia urinaria "International Continence Questionnaireshort form (ICIQ-UI SF). En ambos cuestionarios los valores predictivos están situados muy próximos entre sí.

prueba (21 items del KHQ frente a los 11 del ICIQ-UI SF) y al tiempo de cumplimentación que ya ha sido comentado en otras publicaciones ${ }^{15}$ sino también al porcentaje de casos que se pierden porque no dan respuesta a la totalidad del cuestionario. La insuficiente cumplimentación del cuestionario KHQ en un $7,76 \%$ de los 116 participantes no permitió su asignación a uno de los grupos diagnósticos predeterminado basado en los síntomas; esto ocurrió con el ICIQ-UI SF en el 2,59\% y sólo en el 0,86\% según el estudio urodinámico. Si tenemos en cuenta estos datos, y consideramos que ambas pruebas tienen un valor diagnóstico similar podemos pensar que en la práctica clínica es más aconsejable el uso del ICIQ-UI SF.

En cuanto a las puntuaciones medias obtenidas en el KHQ, éstas evidencian que la dimensión de las relaciones personales es la que menos se ve afectada por el impacto de la IU $(15,96)$. Las dimensiones relativas a relaciones sociales y el descanso y la energía personal tampoco se ven demasiado afectadas $(27,40$ y 27,78 respectivamente). Por el contrario, el impacto en la vida diaria se percibe como importante $(64,1)$. Los valores de las puntuaciones globales $(\mathrm{n}=98)$ tienen como media un valor de 39,94 (DS= 22,11). Estos valo- res totales están muy próximos a los encontrados ya en otros estudios ${ }^{16}$. En el ICQ-UI SF ( $\left.n=101\right)$, la media de los valores totales es de $13,764,11$ ) siendo 4 el valor mínimo y 21 el máximo.

Por otra parte, existe una moderada relación positiva $(0,6, p<0,001)$ entre las puntuaciones totales del KHQ y las del ICIQ-UI SF. Estos valores son muy similares a los encontrados en trabajos publicados fuera de nuestras fronteras ${ }^{16}$. A esto cabe sumar, que también correlacionan de forma significativa las puntuaciones del item 3 del ICIQ-UI SF y el valor global del KHQ $(0,55$, $\mathrm{p}<0,001)$. Por último, hay relación positiva y significativa entre este mismo item 3 del ICIQ-UI SF y la dimensión 2 del KHQ que mide el impacto de la IU en la calidad de vida del paciente tiene de $0,57(p=0,001)$. Estos coeficientes de relación entre las puntuaciones de ambos cuestionarios, nos permiten hablar de una relación significativa, aunque moderada, entre las puntuaciones que se obtienen en ambos cuestionarios.

En un trabajo anterior ${ }^{10}$ se utilizó la misma muestra de pacientes incluida en el presente estudio para conocer el valor del cuestionario ICIQ-UI SF y la prueba de esfuerzo en el diagnóstico diferencial del tipo de IU. Los resultados del presente estudio confirman la utilidad del ICIQUI SF, esta vez comparándolo con el KHQ: parece aconsejable el uso preferencial del ICIQ-UI SF frente al KHQ a la hora de evaluar la IU en el ámbito de la práctica clínica, desde el punto de vista de la paciente.

\section{REFERENCIAS}

1. Abrams P, Cardozo L, Fall M, Griffiths D, Rosier P, Ulmsten U, Van Kerrebroeck P, Victor A, Wein A; Standardisation Sub-Committee of the International Continence Society. The standardisation of terminology in lower urinary tract function: report from the standardisation sub-committee of the international continence society. Urology 2003; 61(1) 37-49.

2. Hannestad YS, Rortveit G, Sandvik H, Hunskaar S. A community-based epidemiological survey of female urinary incontinence: the Norwegian EPINCONT study. J Clin Epidemiol 2000; 53(11):1150-1157.

3. Hunskaar S, Burgio K, Diokno AC, Herzog AR, Hjälmås K, Lapitan MC. Epidemiology and natural history of urinary incontinence in women. Urology 2003; 62 (Suppl 4A):16-23.

4. Hunskaar S, Lose G, Voss S. The prevalence of urinary incontinence in women in four European countries. BJU Int 2004 Feb;93(3):324-330.

5. Jolleys JV, Donovan Jl, Nanchahal K, Peters TJ, Abrams P. Urinary symptoms in the community: How bothersome are they? Br J Urol 1994; 74(5):551-555. 
6. Espuña M. Incontinencia de orina en la mujer. Med Clín (Barc) 2003; 120:464-472.

7. Abrams P, Cardozo L, Khoury S, Wein A. 2005; Incontinence. 3rd International Consultation on Urinary Incontinence. Plymbridge: Mass Health Publication Ltd. 521

8. Badía X, Castro D, Conejero J. Validez del cuestionario King's Health Questionnaire para la evaluación de la calidad de vida de los pacientes con incontinencia urinaria. Med Clin (Barc) 2000; 114: 647-652.

9. Espuña M, Rebollo P, Puig M. Validación de la versión española del ICIQ-IU SF. Un cuestionario para evaluar la incontinencia urinaria. Med Clin (Barc) 2004; 122: 288-292

10. Espuña M, Dilla, T, Castro D, Carbonell C, Casariego J, Puig-Clota M. Analysis of the value of the ICIQ-UI SF questionnaire and stress test in the differential diagnosis of the type of urinary incontinence. Neurouroll Urodyn. 2007 Mar 1; [Epub ahead of prin].

11. Kelleher CJ, Cardozo LD, Khullar V, Salvatore S. A new questionnaire to assess the quality of life of urinary incontinent women. British Journal of Obstetrics and Gynaecology 1997; 104(12):1374-1379.

12. Avery K, Donovan J, Peters TJ, Shaw C, Gotoh M, Abrams P. ICIQ: a brief and robust measure for evaluating the symptoms and impact of urinary incontinence. Neurourol Urodyn 2004; 23(4):322-330.
13. Schäfer W, Abrams P, Liao L, Mattiasson A, Pesce F, Spangberg A, Sterling AM, Zinner NR, van Kerrebroeck P; International Continence Society. Good urodynamic practices: Uroflowmetry, filing cystometry and pressure-flow studies. Neurourol Urodyn 2002; 21(3):261-274.

14. Altman DG. Practical statistics for medical research. London: Chapman \& Hall. 1991; P 409-419.

15. Espuña M, Puig M. Síntomas del tracto urinario inferior de la mujer y afectación de la calidad de vida. Resultados de la aplicación del King's Health Questionnaire. Actas Urol Esp. 2006; 30(7):684-691.

16. Tamanini JTN, Dambros M, D`Ancona CAL, Palma PCR y Netto, NRJr. Validation of the "Internacional Consultation on Incontinente Questionnaire- Short Form" (ICIQ-UI SF) for Portuguese. Rev. Saúde Pública 2004; 38 (3):438-444.

Correspondencia autora: Dra. M. Espuña Pons ICGON. Hospital Clínic

Villarroel 170 - 08036 Barcelona

E-mail autora: 12922mep@comb.es

Información artículo: Original - Incontinencia femenina

Trabajo recibido: febrero 2007

Trabajo aceptado: abril 2007 